\title{
Ground State Energy for Fermions in a 1D Harmonic Trap with Delta Function Interaction
}

\author{
Zhong-Qi $\mathrm{Ma}^{1}$ and C. N. Yang 2 , 田 \\ ${ }^{1}$ Institute of High Energy Physics, Chinese Academy of Sciences, Beijing 100049, China \\ ${ }^{2}$ Tsinghua University, Beijing, China and Chinese University of Hong Kong, Hong Kong
}

(Dated: November 5, 2018)

\begin{abstract}
Conjectures are made for the ground state energy of a large spin $1 / 2$ Fermion system trapped in a 1D harmonic trap with delta function interaction. States with different spin J are separately studied. The Thomas-Fermi method is used as an effective test for the conjecture.
\end{abstract}

PACS numbers: $05.30 . \mathrm{Fk}, 03.75 . \mathrm{Sc}$

There is recent experimental and theoretical interest [1, 2] in a model Hamiltonian for $N$ one dimensional spin $1 / 2$ Fermions in a harmonic trap:

$$
H=\sum_{i=0}^{N}\left[-\frac{1}{2} \frac{\partial^{2}}{\partial x_{i}^{2}}+\frac{1}{2} x_{i}^{2}\right]+g \sum_{i>j} \delta\left(x_{i}-x_{j}\right) .
$$

For $N=2$, the eigenvalue problem of this Hamiltonian had been analytically solved [3]. We concentrate in the present paper on the ground state energy $E_{J}$ for given total spin $J$ as $N \rightarrow \infty$. It is well know from group theory that for spin $J$ the spin wave function is described by the two-row Young pattern $[N-M, M]$ where $M=$ $(N-2 J) / 2$ and the space wave function by its associated pattern 4]. According to a theorem due to Lieb and Mattis [5], when $g$ is finite,

$$
E_{J}<E_{J^{\prime}} \quad \text { if } J<J^{\prime} .
$$

We notice the following facts:

(A) As $g \rightarrow+\infty$, the ground state energy $E_{J}$ for any total spin $J$ approaches [2] a limit

$$
E_{J} \rightarrow \sum_{n=0}^{N-1}\left(\frac{1}{2}+n\right)=\frac{1}{2} N^{2} .
$$

(B) For $g=0$, the ground state wave function for spin $J=N / 2-M$ can be taken to be a product of two determinants [4, 6]:

$$
\begin{aligned}
\Psi & =\operatorname{det}\left[u_{0}\left(x_{1}\right) u_{1}\left(x_{2}\right) \ldots u_{N-M-1}\left(x_{N-M}\right)\right] \\
& \times \operatorname{det}\left[u_{0}\left(x_{N-M+1}\right) \ldots u_{M-1}\left(x_{N}\right)\right]
\end{aligned}
$$

where $u_{i}(x)$ is the normalized eigenfunction of the harmonic oscillator. Its energy is

$$
E_{J}=\frac{1}{2}\left[(N-M)^{2}+M^{2}\right] .
$$

When $J=0, N=2 M$ and $E_{0}=N^{2} / 4$. Equations (2) and (4) indicate that

$$
1 / 4 \leq E_{0} / N^{2} \leq 1 / 2 \quad \text { for } g \geq 0 .
$$

(C) For $g \rightarrow-\infty$, when the total spin $J=0, M=N / 2$ pairs are formed with spatial size of the order of $-g^{-1}$ each. The internal energy of each pair is $-g^{2} / 4$. Thus the $M$ pairs contribute $-M g^{2} / 4$ to the total energy. Between these pairs there are Fermionic repulsion as well as attractive delta function interaction. It is difficult to disentangle this complicated repulsion-attraction mix. But we observe that the Fermionic repulsion in absence of attractive $g$ is given by (5). So it is reasonable to surmise that as $g \rightarrow-\infty$, the repulsion contributes $X$ to $E_{0}$ :

$$
E_{0} \rightarrow-\frac{g^{2}}{4} M+X, \quad \text { as } g \rightarrow-\infty,
$$

where $N^{2} / 2 \geq X \geq 0$. The $X$ term in (6) is small compared with the other term as $g \rightarrow-\infty$. Equation (6) holds for $1 \leq M \leq N / 2$.

Equation (5) shows that for $g \geq 0, E_{0}$ is of order $N^{2}$ while (6) shows that $E_{0}$ is of order $M$ as $g \rightarrow-\infty$. How can that be? This question leads to the following conjecture:

Conjecture 1: As $N=2 M$ and $N \rightarrow \infty$, the $E_{0} / N^{2}$ versus $x=g / \sqrt{N}$ curve approaches a limit:

$$
E_{0} / N^{2} \rightarrow f_{0}(g / \sqrt{N}) .
$$

(21), (44) and (6) show that

$$
\begin{aligned}
& f_{0}(x) \rightarrow 1 / 2 \quad \text { as } \quad x \rightarrow+\infty, \\
& f_{0}(0)=1 / 4, \\
& f_{0}(x) \rightarrow-x^{2} / 8 \quad \text { as } \quad x \rightarrow-\infty .
\end{aligned}
$$

The schematic figure of conjecture is shown in Fig. 1 and its generalization is as follows.

Conjecture 2: For fixed $J / N=b$, the $E_{J} / N^{2}$ versus

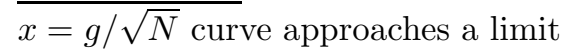

$$
E_{J} / N^{2} \rightarrow f_{b}(g / \sqrt{N}),
$$

where

$$
\begin{aligned}
& f_{b}(x) \rightarrow 1 / 2 \quad \text { as } x \rightarrow+\infty \\
& f_{b}=1 / 4+b^{2}, \\
& f_{b}(x) \rightarrow-\frac{1}{4}\left(\frac{1}{2}-b\right) x^{2} .
\end{aligned}
$$


Simple Test of the Two Conjectures. It seems difficult to prove Conjectures 1 and 2. But there is a very simple but effective test: If Conjectures 1 and 2 are correct, then for large $N$ and given $J$, the slope at $g=0$ is

$$
\frac{d E_{J}}{d g}=N^{3 / 2} \frac{d\left(E_{0} / N^{2}\right)}{d(g / \sqrt{N})} \rightarrow N^{3 / 2} \frac{d f_{b}(t)}{d t},
$$

where $t=g / \sqrt{N}$. Namely, the slope should be proportional to $N^{3 / 2}$ for large $N$.

We calculate $d E_{J} / d g$ at $g=0$ by first order perturbation theory,

$$
\frac{d E_{J}}{d g}=\left\langle\left|\int \psi_{\uparrow}^{\dagger}(x) \psi_{\downarrow}^{\dagger}(x) \psi_{\downarrow}(x) \psi_{\uparrow}(x) d x\right|\right\rangle
$$

where $\psi_{\uparrow}$ and $\psi_{\downarrow}$ are fermionic annihilation operators and the bra \& ket dessignate the ground state at $g=0$. According to (3) this ground state has $N-M$ spin up particles and $M$ spin down particles in single particle states $u_{0}, u_{1}, \ldots$. Thus we have

$$
\frac{d E_{J}}{d g}=\sum_{i=0}^{N-M-1} \sum_{j=0}^{M-1} \int u_{i}^{2}(x) u_{j}^{2}(x) d x .
$$

Defining

$$
S[N-M, M]=\left.N^{-3 / 2} \frac{d E_{J}}{d g}\right|_{g=0},
$$

we have

$$
S[N-M, M]=N^{-3 / 2} \int \rho_{N-M}(x) \rho_{M}(x) d x,
$$

where

$$
\rho_{M}(x)=\sum_{i=0}^{M-1} u_{i}^{2}(x) .
$$

The physical meaning of $\rho_{M}(x)$ is the density of the upspin (or down-spin) particles at $g=0$.

The density $\rho_{M}(x)$ and the slope $S[N-M, M]$ can be calculated in a straightforward way with a computer. We define a scaled density function:

$$
R_{M}(y)=\frac{1}{\sqrt{2 M}} \rho_{M}(\sqrt{2 M} y) .
$$

From (19) we have, as M approaches infinity,

$$
R_{M}(y) \rightarrow 0 \quad|y| \geq 1 .
$$

Fig. 2 shows that $R_{M}(y)$ tends to a limit as $M \rightarrow \infty$ which we shall calculate by the Thomas-Fermi method later in this paper. Here we list in Table 1 values of the slope $S[N-M, M]$ for some given parameter $b=$ $J / N$. One can see from the table that for each value of parameter $b, S[N-M, M]$ rapidly converges to a limit as $\mathrm{N}$ increases, supporting both conjectures 1 and 2.
Table 1 The slope $S[N-M, M]$ for different $b$.

\begin{tabular}{|c|c||c|c||}
\hline \hline$b=0$ & $S[N-M, M]$ & $b=1 / 6$ & $S[N-M, M]$ \\
\hline$[1,1]$ & 0.141047 & {$[2,1]$} & 0.115165 \\
{$[3,3]$} & 0.136147 & {$[4,2]$} & 0.114516 \\
{$[5,5]$} & 0.135539 & {$[6,3]$} & 0.114389 \\
{$[8,8]$} & 0.135292 & {$[8,4]$} & 0.114343 \\
{$[10,10]$} & 0.135228 & {$[10,5]$} & 0.114322 \\
{$[12,12$} & 0.135191 & {$[12,6]$} & 0.114311 \\
{$[15,15]$} & 0.135160 & {$[14,7]$} & 0.114304 \\
{$[18,18]$} & 0.135135 & {$[16,8]$} & 0.114299 \\
{$[19,19]$} & 0.135105 & {$[18,9]$} & 0.114296 \\
{$[20,20]$} & 0.135097 & {$[20,10]$} & 0.114294 \\
\hline Eq.(24) & 0.135095 & Eq.(24) & 0.114284 \\
\hline \hline$b=1 / 4$ & $S[N-M, M]$ & $b=1 / 10$ & $S[N-M, M]$ \\
\hline$[3,1]$ & 0.0935021 & {$[3,2]$} & 0.127119 \\
{$[6,2]$} & 0.0932855 & {$[6,4]$} & 0.126726 \\
{$[9,3]$} & 0.0932444 & {$[9,6]$} & 0.126651 \\
{$[12,4]$} & 0.0932299 & {$[12,8]$} & 0.126624 \\
{$[15,5]$} & 0.0932232 & {$[15,10]$} & 0.126611 \\
{$[18,6]$} & 0.0932195 & {$[18,12]$} & 0.126604 \\
\hline Eq.(24) & 0.0932112 & Eq.(24) & 0.126589 \\
\hline$b=3 / 10$ & $S[N-M, M]$ & $b=1 / 14$ & $S[N-M, M]$ \\
\hline$[4,1]$ & 0.0780554 & {$[4,3]$} & 0.130760 \\
{$[8,2]$} & 0.0779578 & {$[8,6]$} & 0.130501 \\
{$[12,3]$} & 0.0779394 & {$[12,9]$} & 0.130451 \\
{$[16,4]$} & 0.0779330 & {$[16,12]$} & 0.130434 \\
{$[20,5]$} & 0.0779300 & {$[20,15]$} & 0.130429 \\
\hline Eq.(24) & 0.0779247 & Eq.(24) & 0.130411 \\
\hline \hline
\end{tabular}

Thomas-Fermi Method For large values of $M$, we can evaluate $\rho_{M}$ by the Thomas-Fermi method (cf. Fig. $3)$. When $M$ is very large, the particles in $d x$ have as their maximum kinetic energy (=maximum total energy minus the potential) $\sim M-x^{2} / 2$. Thus, the number of particles in $d x$ is $\sqrt{2 M-x^{2}} d x / \pi$. I.e.,

$$
\rho_{M}(x) \rightarrow \sqrt{2 M-x^{2}} / \pi, \quad \text { as } M \rightarrow \infty .
$$

Thus from (20) we have as $M \rightarrow \infty$

$$
R_{M}(y) \rightarrow \sqrt{1-y^{2}} / \pi \quad \text { for }|y| \lesssim 1 .
$$

Considering (20-23), we obtain the slope $S[N-m, M]$ as $M \rightarrow \infty$ from (18) [7]

$$
\begin{aligned}
& \lim _{N \rightarrow \infty} S[N-M, M]=\frac{2 \sqrt{1+2 b}}{3 \pi} \\
& \quad \times\left[F\left(-\frac{1}{2}, \frac{1}{2} ; 1, z\right)-2 b F\left(\frac{1}{2}, \frac{1}{2} ; 1, z\right)\right],
\end{aligned}
$$

where $b=J / N, z=(1-2 b) /(1+2 b)$, and $F$ is the hypergeometric function. For $\mathrm{J}=0$, (24) reduces to,

$$
\lim _{N \rightarrow \infty} S[M, M]=\frac{4}{3 \pi^{2}} \sim 0.135095 .
$$

The numerical values of (24) are listed in the last line for each value $b$ in Table 1 for comparison.

One of the author (ZQM) would like to thank Dr. LiMing Guan for his helpful discussion in drawing Figures. This work was partly supported by the National Natural Science Foundation of China under Grants No. 10675050. 
* Electronic address: cnyang@tsinghua.edu.cn

[1] M. Olshanii, Phys. Rev. Lett. 81, 938 (1998); I. Bloch et al, Rev. Mod. Phys. 80, 885 (2008).

[2] Liming Guan et al, Phys. Rev. Lett. 102, 160402 (2009).

[3] T. Busch et al, Found. Phys. 28, 549 (1998).
[4] C. N. Yang, www.arxiv.org, 0906.4593 (2009). We follow the notation of this paper.

[5] E. Lieb and D. Mattis, Phys. Rev. 125, 164 (1962).

[6] M. D. Girardeau and A. Minguzzi, Phys. Rev. Lett. 99, 230402 (2007).

[7] I. S. Gradshteyn and I. M. Ryzhik, Table of Integrals, Series, and Products, (Academic Press, New York, 1980).

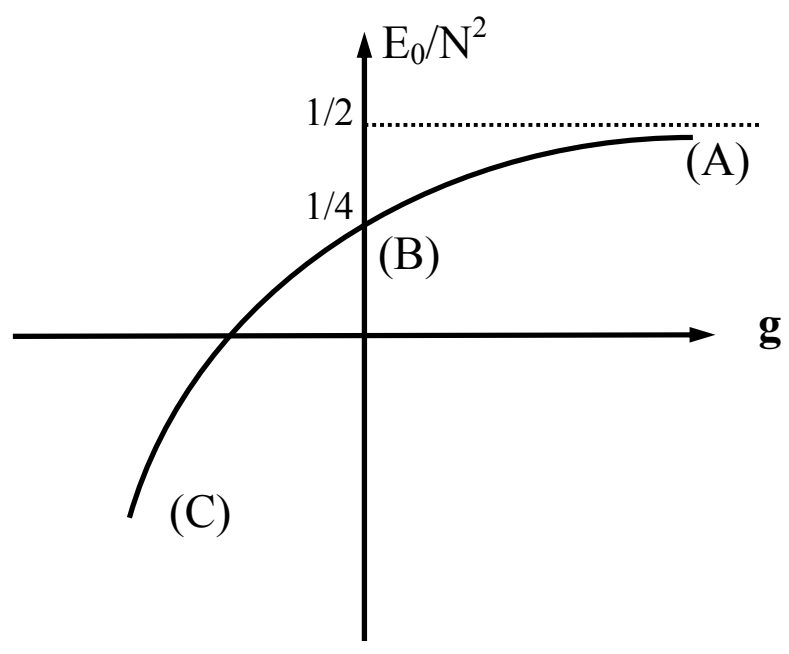

FIG. 1: Ground state energy $E_{J}$ vs. $g$ curve for $J=0$ (Schematic). Equations (8) (9) and (10) refer respectively to regions A, $\mathrm{B}$ and $\mathrm{C}$ in this figure.

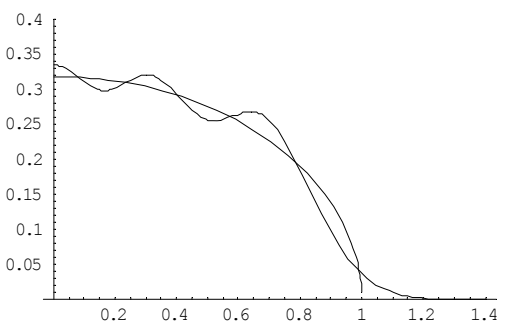

a) $M=5$

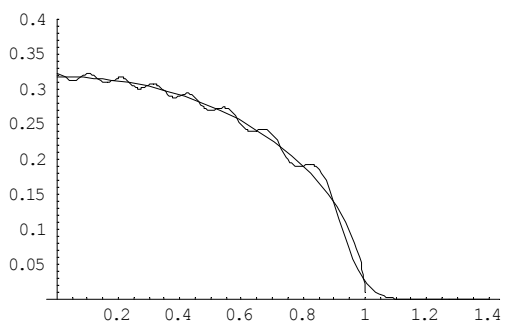

b) $M=15$

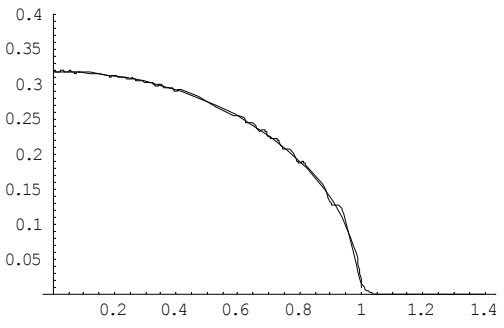

c) $M=55$

FIG. 2: Comparison of $R_{M}(y)$ with its limit $\sqrt{1-y^{2}} / \pi$. 


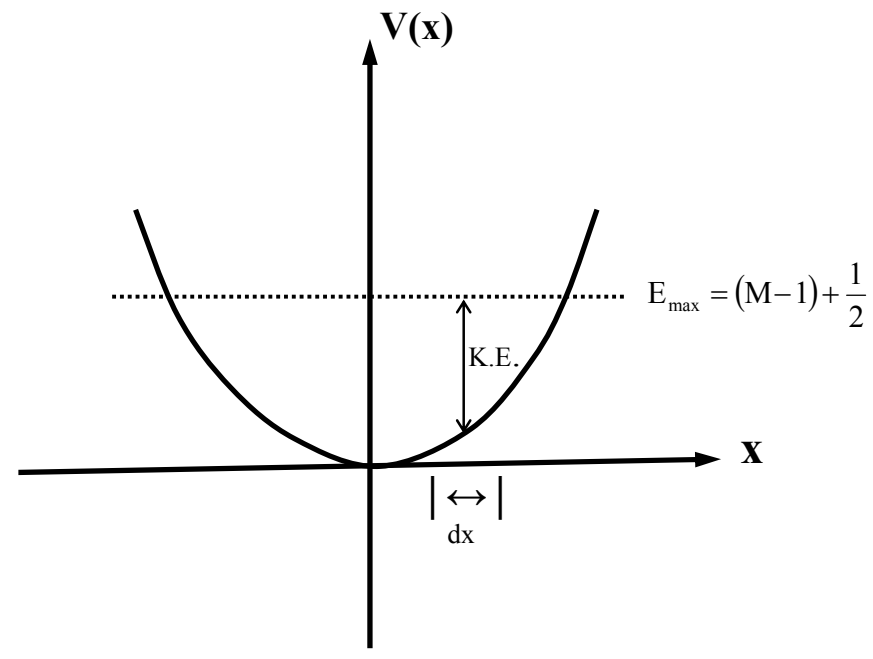

FIG. 3: Thomas-Fermi Method. 